\title{
Revealing genome-wide mRNA and microRNA expression patterns in leukemic cells highlighted "hsa-miR-2278" as a tumor suppressor for regain of chemotherapeutic imatinib response due to targeting STAT5A
}

\author{
Burçin Tezcanlı Kaymaz $^{1}$ - Nur Selvi Günel ${ }^{1}$ - Metin Ceyhan ${ }^{2}$ - Vildan Bozok Çetintaș ${ }^{1}$. \\ Buket Özel $^{1}$ - Melis Kartal Yandım ${ }^{3} \cdot$ Sezgi Kıpçak $^{1}$ • Çağdaș Aktan ${ }^{1}$ • \\ Aysun Adan Gökbulut ${ }^{3}$. Yusuf Baran ${ }^{4}$ - Buket Kosova Can ${ }^{1}$
}

Received: 15 April 2015 / Accepted: 27 April 2015 /Published online: 8 May 2015

(C) International Society of Oncology and BioMarkers (ISOBM) 2015

\begin{abstract}
BCR-ABL oncoprotein stimulates cell proliferation and inhibits apoptosis in chronic myeloid leukemia (CML). For cure, imatinib is a widely used tyrosine kinase inhibitor, but developing chemotherapeutic resistance has to be overcome. In this study, we aimed to determine differing genome-wide microRNA (miRNA) and messenger RNA (mRNA) expression profiles in imatinib resistant (K562/IMA-3 $\mu \mathrm{M}$ ) and parental cells by targeting STAT5A via small interfering RNA (siRNA) applications. After determining possible therapeutic miRNAs, we aimed to check their effects upon cell viability and proliferation, apoptosis, and find a possible miRNA::mRNA interaction to discover the molecular basis of imatinib resistance. We detected that miR-2278 and miR-1245b-3p were most significantly regulated miRNAs according to miRNome array. Upregulating miR2278 expression resulted in the inhibition of resistant leuke-
\end{abstract}

Electronic supplementary material The online version of this article (doi:10.1007/s13277-015-3509-9) contains supplementary material, which is available to authorized users.

Burçin Tezcanlı Kaymaz

burcin.tezcanli@ege.edu.tr; bt1980@gmail.com

1 Medical Biology Department, Ege University Medical School, İzmir, Turkey

2 Oncology Department, 9 Eylül University Medical School, İzmir, Turkey

3 Department of Molecular Biology and Genetics, İzmir Institute of Technology, İzmir, Turkey

4 Faculty of Life and Natural Sciences, Abdullah Gul University, Kayseri, Turkey mic cell proliferation and induced apoptosis, whereas miR$1245 b-3 p$ did not exhibit therapeutic results. Functional analyses indicated that AKT2, STAM2, and STAT5A mRNAs were functional targets for miR-2278 as mimic transfection decreased their expressions both at transcriptional and translational level, thus highlighting miR-2278 as a tumor suppressor. This study provides new insights in discovering the mechanism of imatinib resistance due to upregulating the tumorsuppressor hsa-miR-2278 which stands for a functional therapeutic approach, inhibited leukemic cell proliferation, induced apoptosis, and regain of chemotherapeutic drug response in CML therapy.

Keywords Imatinib resistance $\cdot$ STAT5A $\cdot$ siRNA . Transcriptome and miRNome array $\cdot$ Apoptosis

\section{Introduction}

Chronic myeloid leukemia (CML) is a malignant myeloproliferative neoplasm and exhibits unique biologic character by presence of a reciprocal chromosomal translocation of the Abelson (ABL1) oncogene on 9th chromosome and the breakpoint cluster region (BCR) on 22nd chromosome, as $t(9 ; 22)(\mathrm{q} 34 ; \mathrm{q} 11.2)[1]$. This rearrangement results in the formation of a chimeric fusion oncoprotein defined as p210 kDa BCR-ABL, whereas p185 kDa and p230 kDa proteins can also exist in CML depending upon differences in breakpoints [2]. This encoded BCR-ABL1 is a constitutively activated protein tyrosine kinase caused by excess activation of some famous signaling pathways like RAS/RAF/MAPK, PI3KAkt, JUN, MYC, and Janus kinase/signal transducers and activators of transcription (JAK/STAT) [2, 3]. 
As discovering the molecular basis of CML, new treatment strategies were spread out to block BCR-ABL1' s tyrosine kinase activity like "imatinib" (Glivec ${ }^{\circledR}$, formerly known as CGP 57148B) as being a FDA-approved first-line therapy and, thus, marked an era in CML as a tyrosine kinase inhibitor (TKI) [4]. Although TKI-dependent targeted therapies are generally well tolerated and potentially lifelong, adverse events and gain of resistance are mostly experienced among patients that stand for clinical problems [5]. BCR-ABLdependent and BCR-ABL-independent mechanisms are identified in resistance phenomenon. While overexpression of BCR-ABL and mutations in BCR-ABL are in "dependent group," activation of signaling cascades, overexpression of efflux transporters leading to low TKI levels in the cell, and downregulation of influx transporters that inhibit effective TKI shuttling within the cell are found in "independent group" [6]. Although more potent second- and third-line TKIs were generated, it was reported that imatinib-resistant patients with a defined BCR-ABL mutation also exhibited high risk for development of additional mutations giving also rise to gained resistance to second- or third-line TKI therapies [7]. Thus, identifying new and alternative ways to clarify the underlying resistance mechanism has unique impact for targeted therapy. Such molecular targets are accessible from the defined signaling cascades that play major roles in gained imatinib resistance like STAT5A, from famous JAK/STAT pathway. Since STAT5A has a deep impact upon CML pathogenesis, therefore, the term "STATinib therapies" has ascribed to it [8].

STAT5 activation is correlated with functional cellular processes like cell proliferation, differentiation, migration, apoptosis, and hematopoiesis. BCR-ABL induces a tyrosine phosphorylation that leads to STAT5 dimerization. Dimerized STAT5A enters the nucleus, and then binds to consensus sequences via their DNA binding domain that results in accelerated activation of downstream target genes [9].

Some earlier studies including ours have demonstrated that STAT5A expressional inhibition has therapeutic potential as being an effective molecular target for TKI resistance [10, 11]; thus, the strategy is to target STAT5A in various ways like RNA interference (RNAi) technology.

RNA interference is a sequence-specific gene silencing phenomenon in which small interfering RNAs (siRNAs) trigger messenger RNA (mRNA) transcriptional and posttranscriptional silencing, thus represents therapeutic approach [12]. Besides this, non-coding endogenous microRNAs (miRNAs) regulate gene expression either by causing targetspecific mRNA degradation or translational suppression. Nowadays, miRNAs have become good therapeutic targets since their varying expressions effect cellular fateful events such as regulation of development, differentiation, survival, cell aging, and cell death, whereas their abnormal expression patterns give rise to various diseases - initially cancer and leukemia [13]. In this study, we aimed to determine differing genome-wide miRNA and mRNA expression profiles in imatinib resistant (K562/IMA-3 $\mu \mathrm{M})$ and parental K562 cells indicating CML cell model before and after anti-STAT5A-Chl modified siRNA applications. By this approach, we detected new and candidate miRNAs and mRNAs that were potentially responsible for gained imatinib resistance due to targeting STAT5A. Therefore, new molecular targets-therapeutic miRNAs - could be identified for regaining chemotherapeutic drug response and accelerated cure strategies.

Once we have detected a miRNA that was significantly differentially expressed between resistant and parental cell due STAT5A silencing, we aimed to define the genes that were potentially regulated by that miRNA. Secondarily, we aimed to identify apoptotic cell death ratios due to STAT5A silencing among cells, and also before and after miRNA transfection in order to clarify whether miRNA treatment accelerates leukemic cell apoptosis. At the last stage, we aimed to determine miRNA transfection effects upon cell viability and proliferation. Finally, as following these steps, we tried to understand the molecular basis of gained imatinib resistance a bit more in order to generate clinical benefit and impact, thus put forward new therapeutic approaches.

\section{Materials and methods}

\section{Cell lines and culturing conditions}

Human chronic myelogenous leukemia cell model "K562" was purchased from German Collection of Microorganism and Cell Cultures company. K562 cells were maintained in RPMI 1640 medium containing $10 \%$ heat-inactivated fetal bovine serum (FBS), 100 units of penicillin-streptomycin/ $\mathrm{ml}$, and $1 \% \mathrm{~L}$-glutamine at $37{ }^{\circ} \mathrm{C}$ in humidified air containing $5 \% \mathrm{CO}_{2}$ indicated as "sensitive cells." Imatinib resistant K562 cells were developed by applying increasing doses of imatinib (initial concentration was $50 \mathrm{nM}$ ) for a duration of 2 years. Following each imatinib treatment, subpopulations of living cells were collected and the resultant cells that were resistant to $3 \mu \mathrm{M}$ imatinib are referred to as K562/IMA-3 cells. Cells exhibiting $95 \%$ viability and $80 \%$ confluence were used in experiments.

\section{Gene silencing by siRNA applications}

Gene silencing was achieved due to HiPerFect Transfection Reagent (Qiagen, Valencia, CA, USA) user manual in combination with chemically modified cholesterol conjugated antiSTAT5A siRNA (Dharmacon, Chicago, IL, USA). The cholesterol conjugation resided at the 5 '-end of the siRNA sensestrand that specifically binded to STAT5A mRNA and gene silencing experiments carried out in a duration of $72 \mathrm{~h}$. 


\section{qRT-PCR assay}

In order to confirm gene silencing, STAT5A mRNA transcriptional expression level was determined by real-time quantitative reverse transcriptase polymerase chain reaction (qRTPCR). For this purpose, cells were collected and total RNA was isolated from each sample by MagNA Pure Compact RNA Isolation kit (Roche Applied Science, Mannheim, Germany). Isolated RNAs were reverse transcribed into complementary DNAs (cDNAs) by Transcriptor High Fidelity cDNA Synthesis kit (Roche Applied Science, Mannheim, Germany), and qRT-PCR was performed with gene-specific primers and probes using Fast Start DNA Master Hybridisation Probes and Glucose-6-phosphate dehydrogenase (G6PDH) Housekeeping Gene Set (Roche Applied Science, Mannheim, Germany both) kits in LightCycler 2.0 Instrument (Roche Diagnostics, USA). The relative STAT5A mRNA expression of each sample was calculated by dividing the mRNA copy number of STAT5A to G6PDH mRNA copy number as the reference gene. Gene silencing ratio was determined as comparing untreated control cells' relative STAT5A expression level to siRNA treated cells, and the suppression rate was calculated for both K562 and K562/IMA-3 cells.

\section{Western blot assay}

Another confirmation of silencing was achieved by comparing STAT5A translational expression profile in both siRNA treated and untreated K562 and K562/IMA-3 cells. For this purpose, cells were lysed in $0.1 \mathrm{ml}$ of Complete lysis-M buffer (Roche Applied Science, Mannheim, Germany) kit following the instructions. The protein amounts were calculated by Bradford method. Finally, $45 \mu \mathrm{g}$ of each protein extract was resolved at $8 \%$ SDS-PAGE gel and transferred to PVDF membranes using a dry transfer system iBlot (Invitrogen Corporation, Carlsbad, CA, USA). The used primer antibody concentrations were both 1:1000 diluted STAT5A (06-968, Upstate), STAM2 (\#14674), AKT2 (\#3063), and $\beta$-Actin (\#4967) (for both, Cell Signaling Technology, USA). Primary antibody incubation, blotting, and secondary antibody incubation were done in accordance with iBlot ${ }^{\circledR}$ Western Detection Kit (Invitrogen Corporation, Carlsbad, CA, USA) for chromogenic detection of proteins in iBlot system. The results were evaluated with gel imaging system (Chemi Smart 2000, Vilber Lourmat, Marne-la-Vallée, Cedex, France). Another group of western blot experiments were done in order to determine the interaction between miRNA and its potential target genesmiRNA::mRNA axis of interaction.

\section{Apoptosis assays}

Apoptosis rates of anti-STAT5A-siRNA/cholesterol treated and untreated K562 and K562/IMA-3 cells were quantified by a photometric enzyme immunoassay by using the Cell Death Detection ELISA Kit (Roche Applied Science, Mannheim, Germany) which allows for the detection of mono- and oligonucleosomes and measures apoptotic cell death. For this purpose, $5 \times 104$ cells were collected and kit manual was applied; finally, absorbance was measured spectrophotometrically at $405 \mathrm{~nm}$ with an ELISA reader (Thermo, Vantaa, Finland). Apoptotic cell rates were calculated after the average values of duplicated absorbance measurements of samples were taken and the background value was subtracted from each of these average values. Another group of apoptosis assay was carried out following miRNA treatments in order to determine their apoptotic effects upon cells.

\section{Genome-wide microRNA array (miRNome) and mRNA (transcriptome) array via Agilent 2100 Bioanalyzer}

In order to determine differing genome-wide microRNA and mRNA expression profiles in imatinib resistant (K562/IMA$3 \mu \mathrm{M})$ and parental K562 cells, we used Agilent 2100 Bioanalyzer microarray chip system (Agilent Technologies, Palo Alto, CA). We generated four groups of cells: parental untreated, parental + anti-STAT5A-siRNA transfected, resistant untreated, resistant + anti-STAT5A-siRNA transfected; generating $\times 4$ biological replicates in each group.

\section{RNA isolation, quality, and quantity determination}

Totally, 16 samples' total RNA was isolated via Tripure Isolation Reagent Kit (Roche Applied Science, Mannheim, Germany) and their quality and quantity were determined by Agilent RNA 6000 Nano Kit (Agilent Technologies, Palo Alto, CA) in Agilent 2100 Bioanalyzer. All samples provided $>8.5$ RNA integrity number, a clear gel image, and no DNA contamination was observed on the histogram. We proceeded with microarray PCR.

\section{Genome-wide microRNA array analysis}

Following quality detection steps, 100 ng RNA was reverse transcribed by miRNA Complete Labeling and Hybridization Kit and MicroRNA Spike-In Kit (both Agilent Technologies, Palo Alto, CA) and then labeled with $\mathrm{Cy} 3$ fluorescein dye. Next, these Cy3-labeled cDNAs were hybridized to whole miRNome Human miRNA Microarray, Release 19.0, 8x60K(v19) by using Agilent microRNA Hybridization Kit microarrays for $20 \mathrm{~h}$. Following washing and drying steps with Stabilizing and Drying Solution (Agilent Technologies, Palo Alto, CA) steps, the slides were scanned by Agilent Microarray Scanner. All steps were performed according to the manufacturer (Agilent Technologies, Palo Alto, CA) manuals. By this approach, we analyzed 2042 mature miRNAs from 
miRBase ver:19.0 in $\times 4$ replicates both in resistant and parental cells either anti-STAT5A-siRNA transfected or not.

\section{Genome-wide mRNA array analysis}

Following quality detection steps as indicated above, $100 \mathrm{ng}$ RNA was reverse transcribed by Low Input Quick Amp Labeling Kit, One-color and RNA Spike-In Kit, One-Color (both Agilent Technologies, Palo Alto, CA) and then labeled with Cyanine 3-CTP; thus, fluorescent complementary RNA (cRNA) was generated. Next, we used the RNeasy Mini Kit (Qiagen, Valencia, CA) to purify the amplified cRNA samples and they were hybridized to whole-genome Human GE 8x60K Microarray V2 by using Gene Expression Hybridization Kit (Agilent Technologies, Palo Alto, CA) for $20 \mathrm{~h}$. Following washing and drying steps with Gene Expression Wash Buffer Kit (Agilent Technologies, Palo Alto, CA) steps, the slides were scanned by Agilent Microarray Scanner. All steps were performed according to the manufacturer (Agilent Technologies, Palo Alto, CA) manuals. By this approach, we analyzed 45.033 transcripts in $\times 4$ replicates both in resistant and parental cells either siRNA transfected or not.

\section{Validation steps for selected miRNAs and mRNAs by real-time qRT-PCR}

Following genome-wide microRNA and mRNA array results' evaluation and comparison, we detected that two miRNAs and three mRNAs were significantly regulated due to siRNA treatment among resistant and parental cells. Thus, we validated these expressional changes via qRT-PCR in LightCycler 480.

\section{miRNA validation}

For miRNA validation, 16 total RNA samples $(5 \mathrm{ng} / \mu \mathrm{l}$ amount; totally $25 \mathrm{ng}$ ) were reverse transcribed into cDNA by Universal cDNA synthesis kit (Exiqon, Vedbaek Denmark). In each cDNA synthesis reaction, also cDNA's control was achieved by using synthetic RNA spike-in primers. For qRT-PCR, the obtained cDNAs were 1:80 diluted with nuclease-free water; Snord48 and U6 were used as housekeeping genes. MicroRNA LNA ${ }^{\mathrm{TM}}$ primer sets for hsa-miR1245b-3p and hsa-miR-2278 (Exiqon, Vedbaek Denmark) in cooperation with ExiLENT SYBR ${ }^{\circledR}$ Green Master Mix kit (Exiqon, Vedbaek Denmark) were used in LightCycler 480 II instrument. After PCR, relative quantification of miRNAs were done by $\Delta / \Delta \mathrm{Ct}$ method and the obtained $\mathrm{Ct}$ values or target miRNAs were normalized with housekeeping genes' $\mathrm{Ct}$ values. Thus, microRNA array results were checked once and validated. $m R N A$ validation

For mRNA validation, all 16 total RNA samples $(5 \mathrm{ng} / \mu \mathrm{l}$ amount; totally $55 \mathrm{ng}$ ) were reverse transcribed into cDNA by Transcriptor First Strand cDNA Synthesis Kit (Roche, Applied Science, Mannheim, Germany). In qRT-PCR while UPL probes designed for AKT2, STAM, and STAT5A serve as target genes, GAPDH was used as housekeeping gene, in use with LightCycler ${ }^{\circledR} 480$ Probes Master (Roche, Applied Science, Mannheim, Germany) mix by LightCycler 480 II instrument. The used probes are given in Table 1. Relative quantification of target mRNAs was determined by the same way given above; thus, the mRNA array results were checked and validated.

\section{Transient transfection of miRNA oligonucleotides}

The miRNA mimic or inhibitor sequences specific to hsamiR-1245b-3p and hsa-miR-2278 and also anti-miR miRNA inhibitor (negative control) [all purchased from Ambion (Carlsbad, USA)] were transfected onto cells by HiPerFect Transfection Reagent (Qiagen, Valencia, CA, USA) user manual for a period of $72 \mathrm{~h}$. miRNA transfected cells were used in miRNA expression assay by qRT-PCR, western blot, potentially regulated mRNAs' expression analyses by qRT-PCR, cell proliferation, and apoptosis assays.

\section{Cell viability and proliferation assay}

Cell proliferation assay was performed after transfection of hsa-miR-2278 mimic or hsa-miR-2278 inhibitor. For this purpose, after $72 \mathrm{~h}$ of miRNA transfection, $30 \times 10^{4}$ cells were seeded in 96-well plates, and then, by applying increasing doses of imatinib, cells were incubated for an additional $24 \mathrm{~h}$. Cell viability was determined by using the WST-1 kit (Roche Applied Science, Mannheim, Germany) and absorbance of each sample was measured spectrophotometrically at $450 \mathrm{~nm}$ with an ELISA reader (Thermo, Vantaa, Finland) in triplicates.

\section{Statistical analyses}

The obtained microRNA array and mRNA array images after scanning were analyzed by Agilent Feature Extraction software in order to acquire signals and quality control reports. After passing quality step, the quantile normalization was done via GeneSpring GX (Agilent) 13.0 software. In conclusion, expressional differences within groups were evaluated by Moderated $t$ test and Bonferroni family wise error rate (FWER) for correction and $>2$ or more fold regulation was accepted as significant with $p$ value $\leq 0.05$.

Relative mRNA expression levels gained at the end of qRT-PCR analyses following siRNA treatments, miRNA 
Table 1 The probe sequences used in mRNA expression confirmation analysis in LightCyler 480 II by qRT-PCR

STAM2; UPL\#74

$60 \mathrm{bp}$

F; GCATCCGGCACATTCTGTA

R; GGAGAGCTGCAATTGTTGG

STAT5A; UPL\#110

F; GCATCCGGCACATTCTGTA

R; GGAGAGCTGCAATTGTTGG

AKT2; UPL\#158

F; GTGTTAACAAAACCTCTACCTGTAACC

R; TTCTGGATAATGTATTTCAAACAGGA

expression profiles, and apoptosis assay results were evaluated with Student's $t$ test referring to significance of $p \leq 0.05$ in GraphPad Prism software.

\section{Results}

\section{Targeting STAT5A resulted in expressional downregulation and induced apoptosis}

The efficacy of siRNA transfection was determined by evaluating suppression rates of STAT5A at mRNA and protein levels by RT-qPCR and western blot analysis, respectively. As shown in Fig. 1a, after anti-STAT5A-Chl modified siRNA transfection, STAT5A mRNA expression levels were suppressed by $80.28 \%(-5.02$-fold; $p=0.0015)$ and $91.04 \%$ $(-11.16$-fold; $p<0.0001)$ in K562 and K562/IMA-3 cells, respectively. In accordance with mRNA suppression, also STAT5A protein levels were inhibited due to siRNA treatment in both parental and resistant cells compared to their untransfected counterparts (Fig. 1b).

Next, we determined apoptosis rates following siRNA treatment in both of the cells and indicated that apoptotic cell number was significantly arisen in siRNA transfected cells as compared to their untransfected control cells. As given in Fig. 1c, while K562+ siRNA treated parental group exhibited a 2.2-fold increase $(p<0.0001)$ compared to K562 untransfected cells, and 3.08-fold induction $(p=0.001)$ was detected in K562/IMA-3+ siRNA treated group compared to untransfected K562/IMA-3 cells.

\section{hsa-miR-2278 and hsa-miR-1245b-3p were emphasized due to miRNome array: twinkle to regain of imatinib response}

We analyzed differing genome-wide microRNA expression profiles in K562 and K562/IMA-3 cells, whereas miRNome array results indicated that two miRNAs were significantly regulated among cells due to STAT5A silencing. Since we have generated four groups of cells as K562 UT, K562+ siRNA, K562/IMA-3 UT, and K562/IMA-3+ siRNA, we initially focused on to determine the miRNA that exhibited the highest expressional difference among parental and resistant cells referred as "K562 UT" and "K562/IMA-3 UT." Secondarily, we checked how expression of that miRNA was regulated following siRNA treatment.

We detected that hsa-miR-2278 exhibited the lowest expression pattern in K562/IMA-3 UT cells as 0.028-fold negative regulation $(p=0.047$ ) that stand for a 35.7-fold downregulation compared to K562 UT cells. When we re-checked its expression following siRNA treatment, we detected that it was 1.59 -fold $(p=0.065)$ positively regulated in "K562/IMA-3+ siRNA" cells compared to "K562+ siRNA" that stand for a 56.7-fold upregulation as the initial expression pattern of 0.028 (Supplementary material 1). But a more dramatic expressional change was detected when a comparison was done among K562/IMA-3+ siRNA and K562/IMA-3 UT cells: hsamiR-2278 expression was 10.23 -fold positively regulated ( $p=$ 0.034 ) in K562/IMA-3+ siRNA cells that indicated a 365.3fold increase as compared to the initial expression pattern of 0.028. This expressional regulation following anti-STAT5AsiRNA treatment indicated that resistant cells mimicked sensitive cell expression pattern for hsa-miR-2278.

The maximally expressed one hsa-miR-1245b-3p among resistant and parental cells also showed just the opposite effect following gene silencing, and its expression was 7.14-fold downregulated compared to untransfected resistant cells. So, hsa-miR-1245b-3p expression was also converged to parental cells due to STAT5A silencing with 146-fold downregulation as its initial expression (Supplementary material 1).

Another important point is the need for validation of these expressional changes once again via qRT-PCR since they were detected by chip system. So, next, we re-quantitated expression patterns of hsa-miR-2278 and hsa-miR-1245b-3p. Similar results were detected for miRNA expression profiles. While hsamiR-2278 was 0.068 -fold negatively regulated $(p=0.029)$ in K562/IMA-3 UT compared to K562 UT cells in means of 14.7-fold downregulation in resistant untreated cell (Fig. 2a); when anti-STAT5A-siRNA was transfected, the expression was 10.02-fold positively regulated $(p<0.0001)$ in K562/IMA-3+ siRNA cells compared to K562/IMA-3 UT cells (Fig. 2b). This means that, following siRNA treatment in resistant cells, hsamiR-2278 expression was upregulated compared to its initial expression and exhibited more similar expression pattern as in parental untransfected cells.

For hsa-miR-1245b-3p validation analyses, similar results were obtained. While 20.8-fold upregulation ( $p<0.0001)$ was detected in K562/IMA-3 cells compared to parental cells (Fig. 2c), when anti-STAT5A-siRNA was transfected, the expression was 18.07-fold negatively regulated $(p<0.0001)$ in K562/IMA-3+ siRNA cells compared to K562/IMA-3 UT cells (Fig. 2d).

All these data pointed out that silencing STAT5A had totally changed expression patterns of hsa-miR-2278 and hsa- 
Fig. 1 Targeting STAT5A resulted in expressional downregulation and induced apoptosis: a STAT5A mRNA expression levels were downregulated by 5.02 -fold and 11.16-fold in K562 and K562/ IMA-3 cells, respectively $\left({ }^{*} p=0.0015,{ }^{*} p<0.0001\right)$. b The level of STAT5A protein expression was decreased by siRNA transfection in both K562 and K562/IMA-3 cells. Beta-actin was used as loading control, \#SM1811, Fermentas Protein marker was used in gels. c Apoptosis induction was detected in both siRNA treated cells with 2.2-fold in $\mathrm{K} 562+$ siRNA parental group and 3.08-fold in K562/IMA-3+ siRNA resistant group compared to their untransfected counterparts $\left({ }^{*} p<0.0001,{ }^{* *} p=0.001\right)$ a

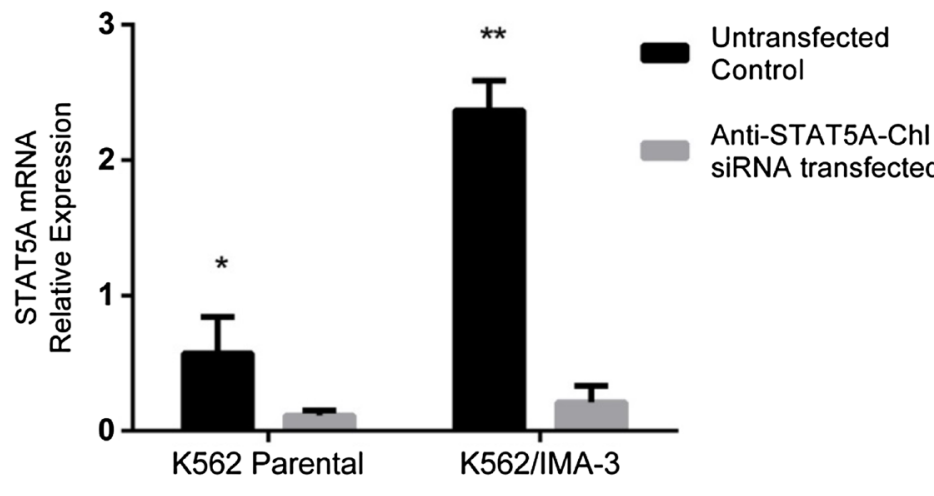

b

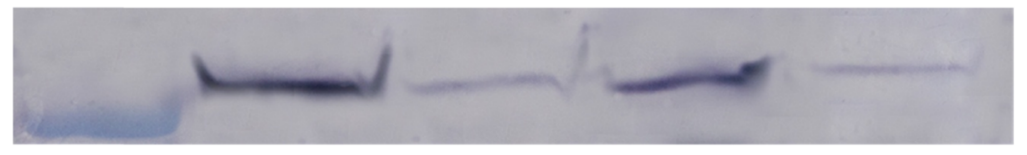

STAT5A, $96 \mathrm{kDa}$

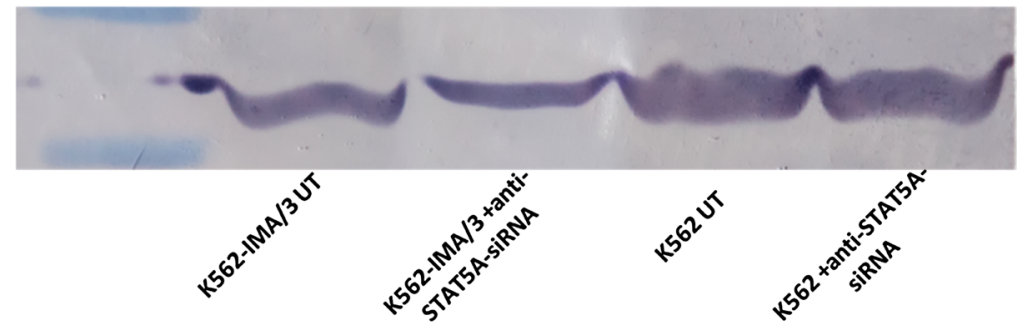

B-Actin , $45 \mathrm{kDa}$

\section{C}

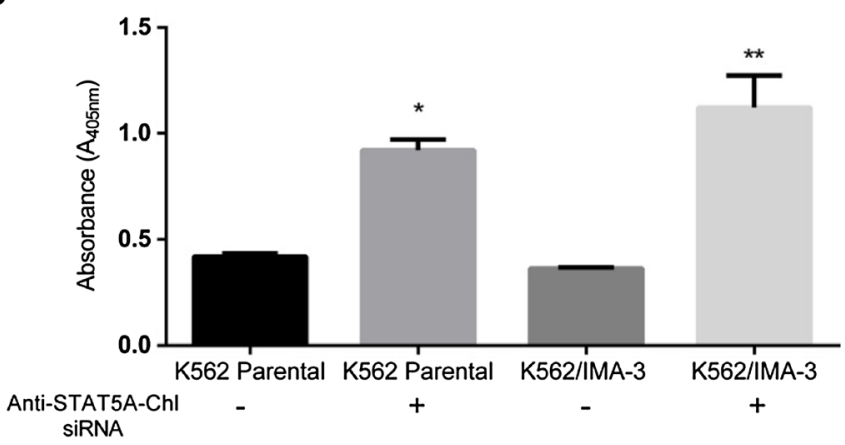

miR-1245b-3p in resistant cells, whereas resistant cells started to exhibit similar expression pattern as sensitive cells which might have important clinical reflection such as regain of nilotinib response.

\section{Genome-wide mRNA array results: combined analysis in regard of detecting hsa-miR-2278 and hsa-miR-1245b-3p regulated genes}

We used genome-wide mRNA array results for two outcomes. First, we aimed to determine the differing expression patterns among parental and resistant cells before and after siRNA treatment. We detected some critical data in comparison between K562/IMA-3+ siRNA cells and K562/IMA-3 UT cells. As for the array results, MDM2 oncogene expression was 3.76-fold significantly upregulated $(p=0.014)$ in resistant untransfected cells which meant that silencing STAT5A had led to expressional downregulation in resistant cells. Another promising result was seen in RAS protein activator like 1 (GAP1 like) (RASAL1), as 3.42-fold significant increase $(p=0.0039)$ in resistant untransfected cells compared to their siRNA transfected outcomes. Also, STAT5A expression levels were decreased in both siRNA transfected groups, with 4.34-fold $(p=0.0021)$ and 2.8-fold $(p=0.032)$ inhibitions in K562/IMA-3+ siRNA and K562+ siRNA cells, respectively, compared to their untransfected counterparts (Supplementary material 2).

Secondarily and more importantly, we used these wholegenome mRNA array data after determining potential target mRNAs for either hsa-miR-2278 or hsa-miR-1245b-3p in 

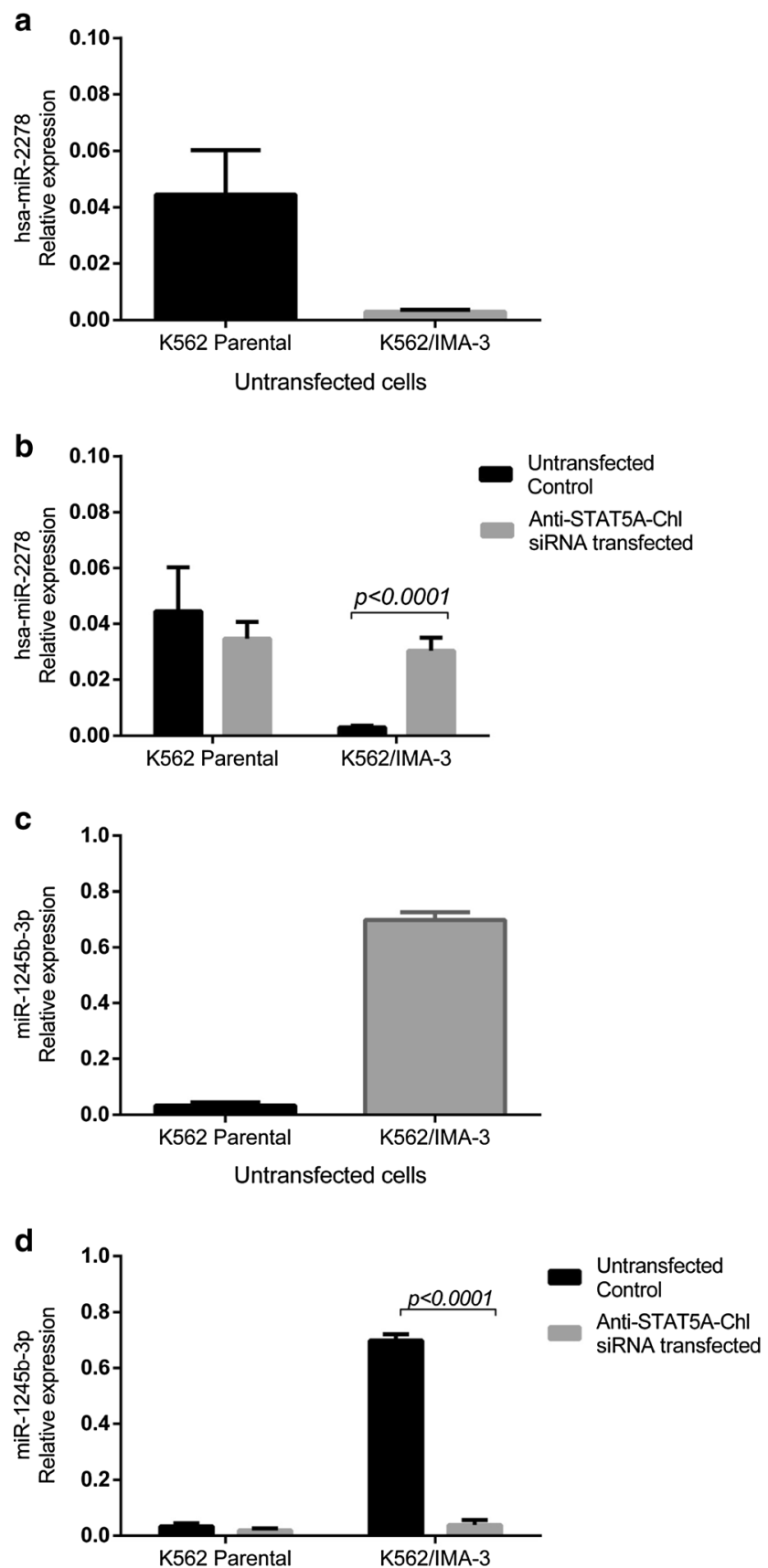

Fig. 2 Validation results by qRT-PCR for hsa-miR-2278 and hsa-miR$1245 \mathrm{~b}-3 \mathrm{p}$ expressional changes due to siRNA applications obtained from miRNA array. a hsa-miR-2278 expression was 14.8-fold upregulated in K562 parental cells compared to K562/IMA-3 resistant cells $(p=0.029)$. b anti-STAT5A-siRNA treatment changed hsa-miR-2278 expression pattern in resistant cells, and its expression was 10.02-fold upregulated $(p<0.0001)$ compared to its untransfected counterpart. $\mathbf{c}$ hsa-miR-1245b$3 p$ expression was 20.8-fold upregulated $(p<0.0001)$ in K562/IMA-3 cells compared to parental K562 cells. d Following anti-STAT5AsiRNA treatment, hsa-miR-1245b-3p expression was 18.07-fold downregulated $(p<0.0001)$ in K562/IMA-3 cells compared to its untransfected counterpart

correlation with miRTar in silico analyses and to prove the interaction between them with further functional analyses.
But in miRTar-based interaction search, no potential regulation was detected between either hsa-miR-2278:MDM2 or hsa-miR-2278:RASAL1 and hsa-miR-1245b-3p:MDM2 or hsa-miR-1245b-3p: RASAL1 except STAT5A.

At this point, we focused on the genes that were found in JAK/STAT pathway as STAT5A being a member of this pathway and our previous experiences with this cascade in CML. After determining potential target genes by in silico analyses, we checked their expression profiles once from wholegenome mRNA array and proceeded with further analyses as given below to prove the interactions.

Searching for potential targets of hsa-miR-2278 and hsa-miR-1245b-3p: STAT5A, AKT2, and STAM2 axis of interaction for hsa-miR-2278

In further analyses, we explored the target genes that hsa-miR2278 and hsa-miR-1245b-3p might regulate; thus, we examined the predicted targets of these miRNAs using the miRTar and TargetScan in silico algorithms. The analysis predicted that there are complementary sites that recognize the seed region of hsa-miR-2278 on 23 genes' ( $A K T 2, C B L, C C N D 2$, CCND3, CSF2, CSH1, GH1, GH2, IFNAR1, IL2RB, IL15RA, LEPR, OSM, PIM1, PIK3R1, STAM2, STAT2, STAT5A, STAT6, TPO, PIAS2, IL22RA2, IL28RA) mRNA 3' UTR from the JAK/STAT pathway, whereas miR-1245b-3p exhibited none. Among them, STAT5A, AKT2 (v-akt murine thymoma viral oncogene homolog 2), and STAM2 (signal transducing adaptor molecule) were selected for also taking place in the whole-genome mRNA array obtained data. We selected STAT5A as being the key player of the study, AKT2 for being an oncogene and STAM2 as being a signal transducer of the pathway. Functional analyses were carried out in order to confirm regulation between hsa-miR-2278 and STAT5A, AKT2 and STAM2 axis, and also to figure out the possible interaction of these genes for miR-1245b-3p.

At this point, before ongoing with transient transfection of miRNA oligonucleotides onto cells in order to confirm the potential regulation between them, we checked STAT5A, AKT2, and STAM2 mRNA expression values from the whole-genome mRNA array data. We detected that both of the mRNA expressions were downregulated by 4.34 -fold ( $p=$ $0.0021), 3.78$-fold $(p=0.037)$, and 3.42-fold ( $p=0.043)$, respectively, following siRNA treatment in resistant cells compared to their untransfected counterparts (Supplementary material 2).

Next, we validated these genes' expressions by qRT-PCR in order to confirm whole-genome mRNA array result. We observed similar results and detected that while STAT5A was 3.41-fold $(p<0.0001)$ decreased, AKT2 was 3.15-fold $(p<0.0001)$ and STAM2 was 2.98 -fold $(p=0.0002)$ downregulated in siRNA treated resistant cells compared to 
untransfected resistant cells (Fig. 3) in re-quantification of genes via real-time qRT-PCR in LightCycler 480.

By this approach, we acquired a beneficial clue and supportive primary data that hsa-miR-2278 might be a tumor suppressor before ongoing with miRNA transfection. Since its expression was dramatically downregulated in resistant cells, the critical intervention as STAT5A gene silencing increased its expression, and this upregulation triggered the cells to display as sensitive cell expression pattern which led us to speculate that it could be a tumor suppressor at the early stages of the study.

\section{Effects of miRNA oligonucleotide transfections upon imatinib resistance}

\section{Transfection efficiency control}

Prior to studying the effects of hsa-miR-2278 and hsa-1245b$3 p$, we used mimic or inhibitor miRNAs in order to check transfection efficiency before ongoing with further experiments. While expression levels of hsa-miR-2278 and hsamiR-1245b-3p were significantly increased by 13.1 -fold $(p<0.0001)$ and 2.92-fold $(p<0.0001)$, their expressions were decreased by 3.11 -fold $(p<0.0001)$ and 2.75 -fold $(p=0.0001$, respectively, following transfection either with mimic or inhibitor miRNAs, respectively (Fig. 4a, b), suggesting that the oligonucleotides were efficiently transfected onto cells and thus enhanced or repressed the expression of miRNAs.

\section{Effects of miRNA transfection upon cell viability and proliferation}

Initially, we analyzed cell proliferation difference between parental and resistant cells due to imatinib treatment and detected that cell viability was reduced in a dose-dependent manner. While $\mathrm{IC}_{50}$ dose of imatinib was calculated as $282 \mathrm{nM}$ for K562 parental cells, it was calculated as $12.54 \mu \mathrm{M}$ for K562/IMA-3 resistant cells (Fig. 5a). Next, we investigated the effects of hsa-miR-2278 and hsa-miR-1245b-3p mimic or inhibitor transfection upon viability and proliferation of leukemia cells; we detected that upregulation in the expression level of hsa-miR-2278 in resistant cells induced inhibition of leukemic cell proliferation, which in turn reduced $\mathrm{IC}_{50}$ dose to $3.62 \mu \mathrm{M}$, and the average cell viability was reduced to $7.12 \pm$ $0.66 \%$ in $20 \mu \mathrm{M}$ imatinib treated resistant cells compared to NC inhibitor miRNA treated resistant cells (Fig. 5b). After anti-miR-1245b-3p transfection, $\mathrm{IC}_{50}$ dose of imatinib was reduced to $5.51 \mu \mathrm{M}$ and the average cell viability was decreased to $14.21 \pm 0.79 \%$ in $20 \mu \mathrm{M}$ imatinib treated resistant cells compared to NC inhibitor miRNA treated resistant cells (Fig. 5c).

Taken together, these results indicated that especially premiR-2278 transfection and to a lesser content anti-miR$1245 \mathrm{~b}-3 \mathrm{p}$ transfection increased sensitivity to imatinib in resistant cells. While pre-miR-2278 transfected resistant cells became 3.46 times more sensitive to imatinib, and anti-miR1245b-3p transfection exhibited 2.28-fold increased sensitivity compared to their NC inhibitor miRNA transfected counterparts.

\section{Increased expression levels of hsa-miR-2278 resulted} in induced leukemic cell apoptosis

As demonstrated in Fig. 6, while apoptosis rate was 5.06-fold upregulated $(p<0.0001)$ in pre-miR-2278 transfected resistant cells compared to untreated resistant cells, anti-miR-1245b-3p transfection triggered 1.92-fold induced apoptosis $(p=0.002)$ compared to untreated group in resistant cells. These findings suggested that apoptosis induction of imatinib resistant cells was significantly enhanced especially via upregulating hsamiR-2278 expression, which in turn resulted in regain of chemotherapeutic response.

\section{AKT2, STAM2, and STAT5A are functional targets of hsa-miR-2278}

At the last stage of the study, we aimed to determine the possible interaction of STAT5A, AKT2, and STAM2 mRNAs

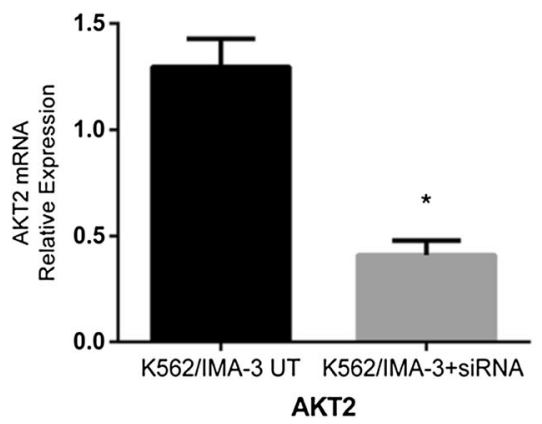

Fig. 3 Validation results by qRT-PCR for STAT5A, AKT2, and STAM2 mRNA expressional changes due to siRNA applications obtained from mRNA array: While STAT5A expression was 3.41 -fold $(* * p<0.0001)$ decreased, AKT2 expression was 3.15 -fold $\left({ }^{*} p<0.0001\right)$ downregulated
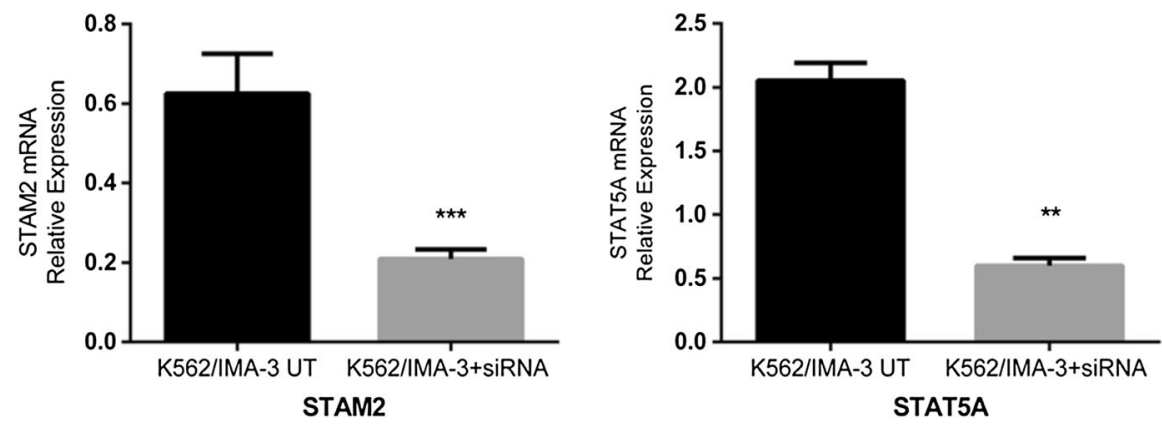

and STAM2 expression was 2.98 -fold $(* * * p=0.0002)$ downregulated in siRNA treated K562/IMA-3 resistant cells compared to untransfected K562/IMA-3 resistant cells after quantification of mRNAs by real-time qRT-PCR in LightCycler 480 

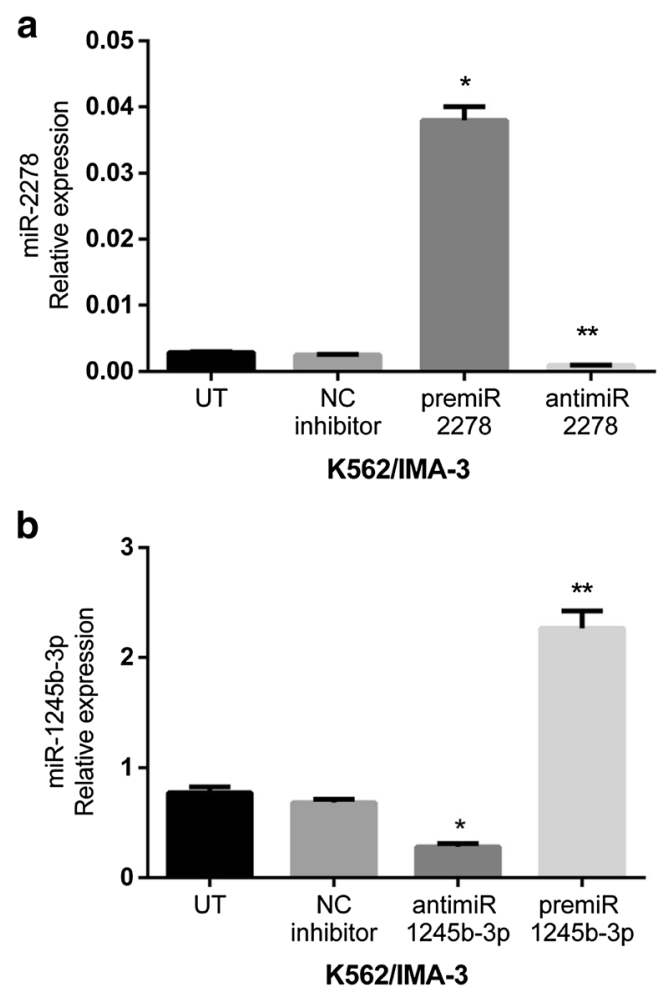

Fig. 4 The expression levels of hsa-miR-2278 and hsa-miR-1245b-3p following mimic or inhibitor treatment: a While hsa-miR-2278 expression was increased by 13.1 -fold $\left({ }^{*} p<0.0001\right)$, it was decreased by 3.11 -fold $(* * p<0.0001)$ following mimic and inhibitor miRNA transfections, respectively. b While hsa-miR-1245b-3p expression was 2.92 -fold upregulated $(p<0.0001)$, it was decreased by 2.75 -fold ( $p=$ $0.0001)$ following transfection either with mimic or inhibitor, respectively

between either with hsa-miR-2278 or hsa-miR-1245b-3p with functional analysis. At the early stages of the study, we have detected that the following anti-STAT5A-siRNA treatment STAT5A, AKT2, and STAM2 mRNA expression levels were downregulated in chip format and these results were validated via qRT-PCR. Then, we had aimed to gain similar effects as siRNA treatment with significantly differing miRNAs as hsamiR-2278 and hsa-miR-1245b-3p either by applying mimic or inhibitor oligonucleotides.

Initially, we determined differing expression profiles of these target mRNAs between parental and resistant cells by qRT-PCR. As given in Fig. 7a, we detected that each mRNA expression exhibited a higher expression pattern in K562/ IMA-3 cells compared to parental cells. While AKT2 expression was 1.87-fold higher, STAM2 and STAT5A mRNA expressions were 1.76-fold and 3.10-fold upregulated in resistant cells, $p<0.0001$ for each. Next, we analyzed their expressional changes following miRNA transfections. We observed that while pre-miR-2278 transfected resistant cells triggered 1.89-fold expression downregulation in AKT2 mRNA expression in resistant cells compared to NC inhibitor miRNA transfected cells, STAM2 was 1.81-fold and STAT5A expression was 4.78 -fold downregulated ( $p<0.0001$ for each), whereas anti-miR-2278 exhibited no significant effect (Fig. 7b). We made similar analyses for hsa-miR-1245b-3p and did not observe significant differences neither mimic nor inhibitor transfections for STAT5A, AKT2, and STAM2 mRNA expression profiles in resistant cells (Fig. 7c).

Next, we checked these potential target mRNAs' varying translational expression due to miRNA transfections by western blot analysis. The obtained results were in the same line with mRNA results. While pre-miR-2278 oligonucleotide transfection efficiently downregulated AKT2, STAM2, and STAT5A protein expressions, miR-1245b-3p mimic or inhibitor transfection did not affect their protein expression levels (Fig. 7d).

These results indicated that STAT5A, AKT2, and STAM2 were not regulated by hsa-miR-1245b-3p. Already, no interaction was reported between hsa-miR-1245b-3p and these mRNAs by in silico analyses, but we had preferred to proceed with further analyses in case there could be. But we detected that STAT5A, AKT2, and STAM2 mRNAs were regulated by hsa-miR-2278, and this miRNA was probably a tumor suppressor thus upregulating its expression resulted in inhibition in transcriptional and translational downregulation in its potential target genes.

\section{Discussion}

In this study, we have selected the headliner as STAT5A since we have experienced before that STAT5A exhibited major effects upon the molecular basis of hematological malignancies and gain of imatinib resistance; thus, targeting STAT5A had remarkable clinical benefit as regain of chemotherapeutic response and induced leukemic cell apoptosis especially in imatinib resistant cells [11]. Based upon our previous studies, in this current study, STAT5A was the main focus of our interest and our selected target. Similar to us, Wang $\mathrm{H}$ et al. also reported that upregulated activation of JAK/STAT signaling pathway had promoted proliferation of leukemic cells in CML, whereas STATs were therapeutic targets then [14].

We analyzed genome-wide microRNA and mRNA expression patterns as 2042 mature miRNAs from miRBase ver:19.0 and 45.033 transcripts in parental K562 and imatinib resistant K562/IMA-3 cells before and after silencing of STAT5A by siRNA applications. As for the obtained results following miRNome array, we detected that 273 miRNA expressions were significantly upregulated ranging between 1047.86-fold and 2.35-fold, whereas 131 miRNAs were significantly downregulated between -2.56 -fold and -35.7-fold in resistant cells compared to sensitive cells. We detected that among them, while hsa-miR-1245b-3p exhibited the highest expression, hsa-miR-2278 showed the lowest expression in resistant cells compared to parental cells. In literature search, till now, there are no published papers related with hsa-miR-1245b-3p that 

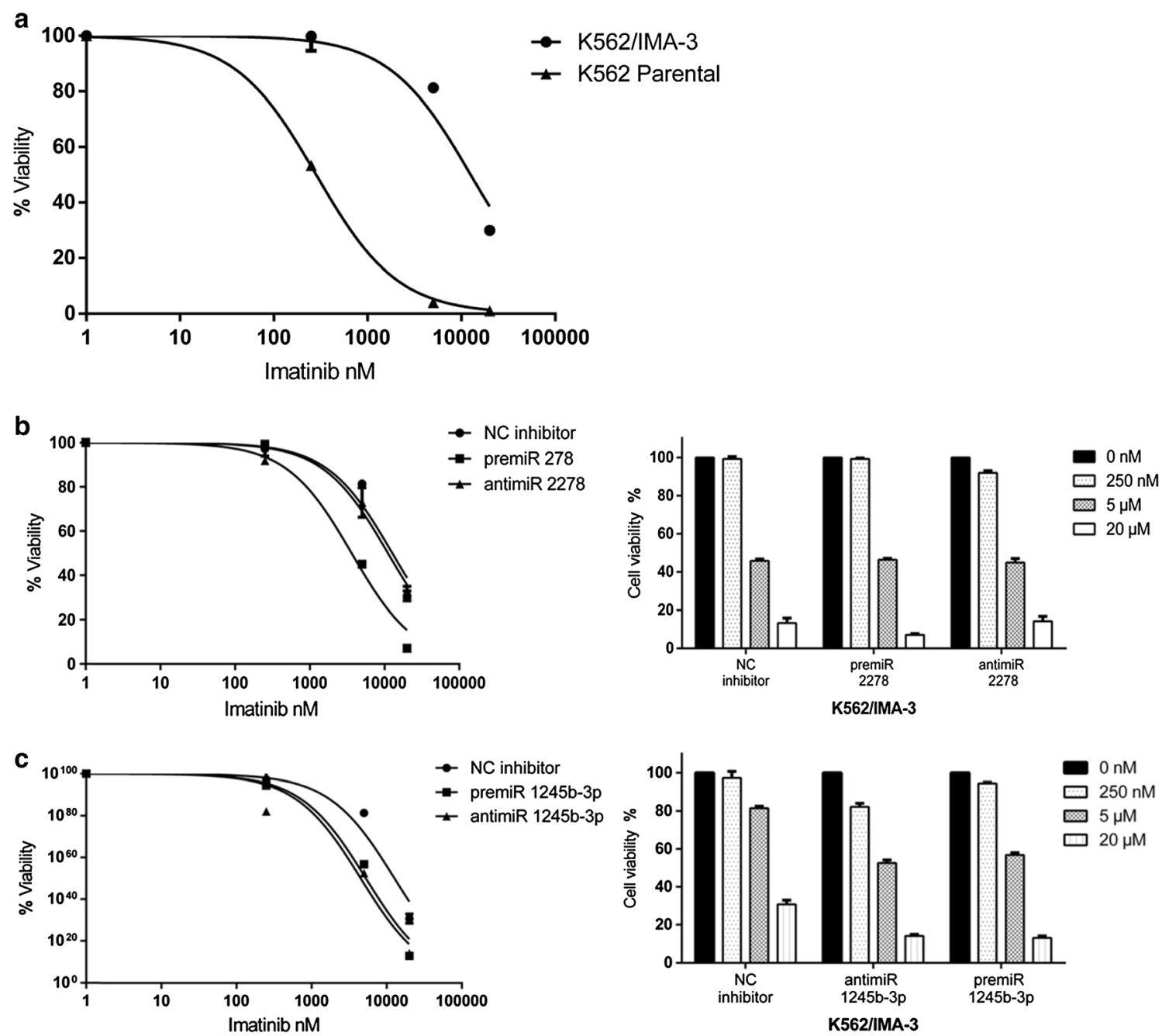

Fig. 5 Effects of miRNA transfection upon cell viability and proliferation: a Differing $\mathrm{IC}_{50}$ doses of imatinib in K562/IMA-3 resistant and $\mathrm{K} 562$ parental cells; calculated as $12.54 \mu \mathrm{M}$ and $282 \mathrm{nM}$, respectively. b pre-miR-2278 transfection inhibited resistant leukemic cell proliferation in a dose-dependent manner and $\mathrm{IC}_{50}$ value was recalculated as $3.62 \mu \mathrm{M}$ (left side) and cell viability was reduced to

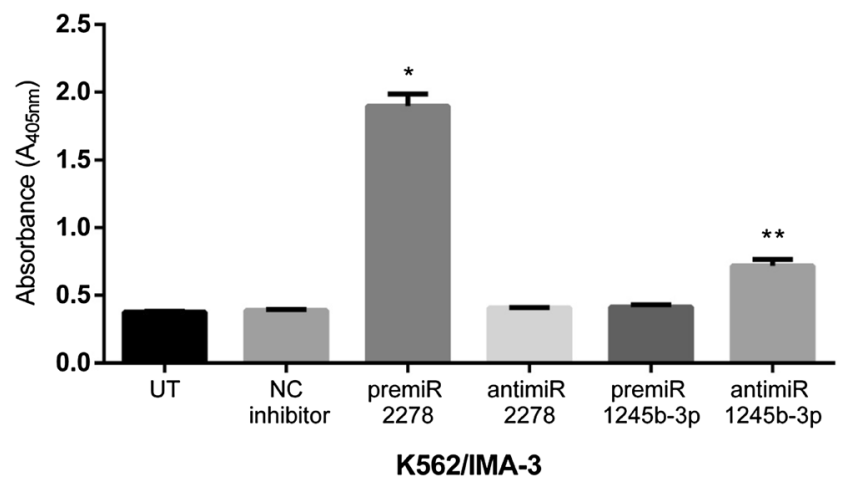

Fig. 6 Increased expression levels of hsa-miR-2278 resulted in induced leukemic cell apoptosis: While pre-miR-2278 transfection triggered 5.06fold upregulated $\left({ }^{*} p<0.0001\right)$ apoptosis induction, anti-miR-1245b-3p transfection induced 1.92 -fold increased $(* * p=0.002)$ apoptosis in resistant cells both compared to control cells
$7.12 \pm 0.66$ with $20 \mu \mathrm{M}$ imatinib treatment (right side). c anti-miR$1245 b-3 p$ transfection triggered a lesser leukemic cell proliferation inhibition, whereas $\mathrm{IC}_{50}$ value of imatinib was reduced to $5.51 \mu \mathrm{M}$ (left side) and cell viability was reduced to $14.21 \pm 0.79 \%$ with $20 \mu \mathrm{M}$ imatinib treatment (right side)

stands for the maximally expressed miRNA in our imatinib resistant cells. Whereas only one paper for hsa-miR-2278 could be found, which was in a totally different topic as a SNP analyses (rs28366) for IL22RA2 and to define whether hsa-miR-2278 binding affinity was significantly affected by this SNP [15].

But there are some recently published papers in regard to investigating miRNome transcriptome in hematologic cancers as chronic myeloid leukemia [16-18]. Among them, in Bhutra et al.' s study, genome-wide miRNA expressions were profiled in K562 cells following imatinib treatment, and miR493-5p was reported as the most significantly regulated miRNA among treated and untreated control cells with 1.52fold upregulation in its expression due to imatinib treatment. We have also a result for miR-493-5p, and similar to their 
Fig. 7 AKT2, STAM2, and STAT5A are functional targets of hsa-miR-2278: a Differing expression patterns of STAT5A, AKT2, and STAM2 mRNAs between K562/IMA-3 resistant and parental cells. While AKT2 expression was 1.87 -fold higher, STAM2 and STAT5A mRNA expressions were 1.76-fold and 3.10-fold upregulated in resistant cells, $* * * * * * p<0.0001$ for each b AKT2, STAM2, and STAT5A mRNA expression levels in K562/IMA-3 resistant cells after miRNA transfection was requantitated by qRT-PCR. While expressional down regulations were detected by pre-miR-2278, increases were observed following anti-miR-2278 treatment for each mRNA compared to $\mathrm{NC}$ inhibitor treated group $(p<0.0001$ for all). $\mathbf{c}$ antimiR-1245b-3p transfection did not cause any significant expressional changes in mRNA profiles regarding AKT2,

STAM2, and STAT5A are not potential targets of has-miR1245b-3p. d The level of AKT2, STAM2, and STAT5A protein expression levels was decreased following pre-miR-2278 transfection, whereas anti-miR1245b-3p did not affect any. Betaactin was used as loading control, \#SM1811, Fermentas Protein marker was used in gels
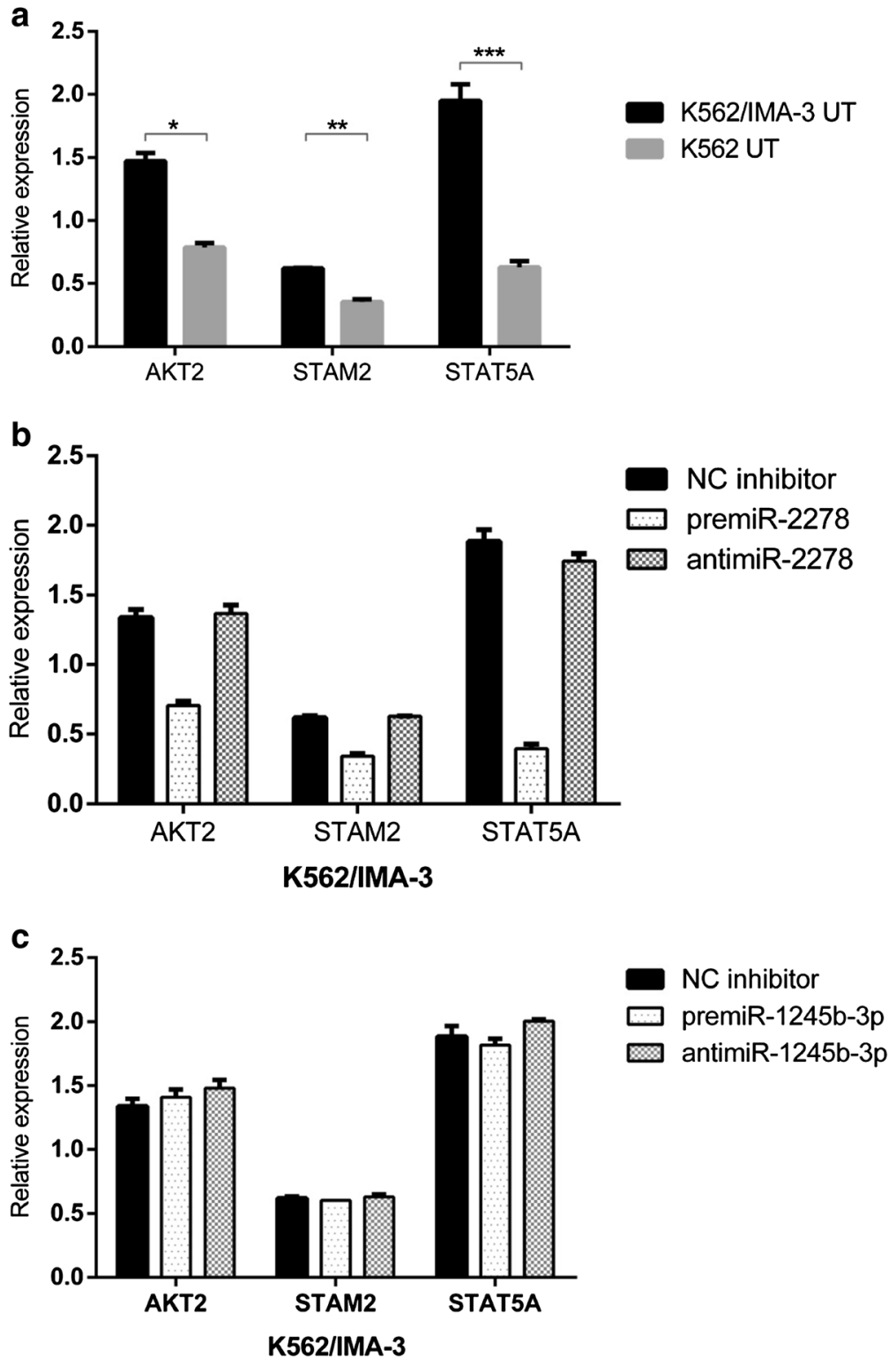

d

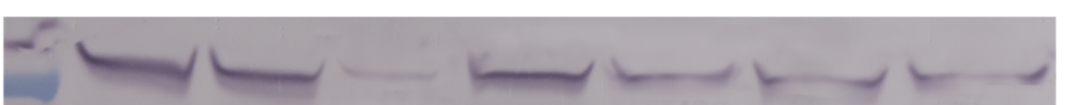

STAT5A, $96 \mathrm{kDa}$

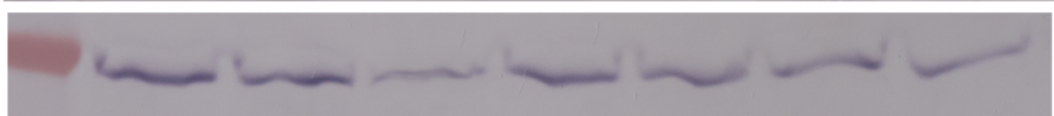

STAM2, $70 \mathrm{kDa}$

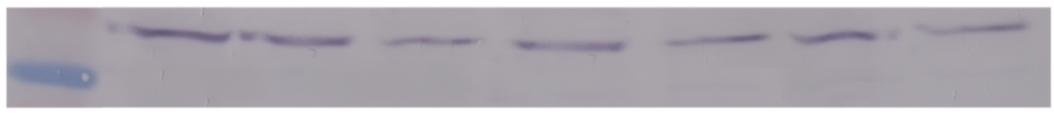

AKT2, $60 \mathrm{kDa}$

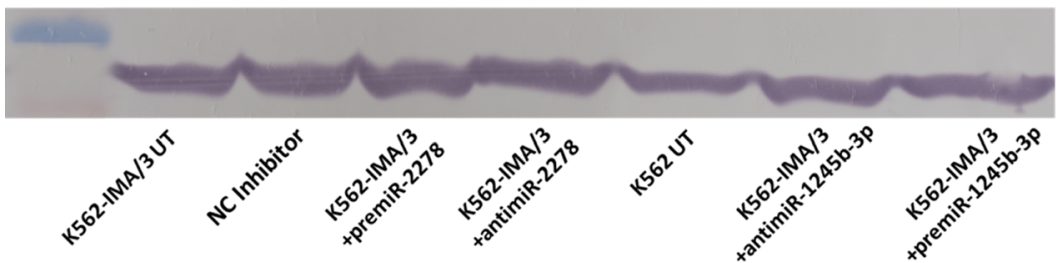

B-Actin , $45 \mathrm{kDa}$ 
reports, we have detected that it was 3.54-fold upregulated in imatinib resistant cells compared to parental cells.

Based upon the enlargement of analyzed patterns, our study seems to be the first one that handled genome-wide screening as for both miRNA and mRNA, in point of silencing STAT5A, thus comparing imatinib sensitive and resistant cells which stands for the superiority of our study. Following siRNA treatments, we detected that hsa-miR-2278 expression was significantly upregulated in its untransfected counterpart resistant cells. So, the minimal expression pattern was 365.3fold increased following silencing STAT5A, and the gained expression profile was similar to sensitive parental cells, which led us to conclude that resistant cells exhibited sensitive cells' expression pattern. Similar expressional differentiation due to siRNA treatment was observed in hsa-miR-1245b-3p, and its expression was more likely to parental cells afterwards.

After performing validation steps for these expressional changes with qRT-PCR analyses, we continued with functional analyses in order to define regulation between these miRNAs and their potential target mRNAs. Due to wholegenome mRNA array data and using in silico algorithms, we detected that STAT5A, AKT2, and STAM2 from JAK/STAT pathway were potential target mRNAs for has-miR-2278 by applying mimic transfection specific to hsa-miR-2278. The obtained results from qRT-PCR assay and western blot analyses indicated that while pre-miR-2278 transfection led expressional decreases in STAT5A, AKT2, and STAM2 mRNA and protein expression levels that normally exhibit increased expression profiles in CML pathogenesis [19]. Thus, we predicted that hsa-miR-2278 was probably a tumor suppressor while its upregulated expression had therapeutic effect upon resistant cells. But no interaction was reported for hsa-miR$1245 \mathrm{~b}-3 \mathrm{p}$ and these selected mRNAs, and we also did not find any at the end of miRNA transfections and functional analyses. But downregulation in miR-1245b-3p expression exhibited some promising results as sensitizing cells to imatinib by 1.66-fold and inducing apoptosis 1.92-fold in resistant cells, so we speculated that miR-1245b-3p displayed these naive therapeutic responses by targeting some other mRNAs.

Also, we checked miRNA transfection effect upon cell viability and proliferation and detected that leukemic cell proliferation was dramatically reduced following pre-miR-2278 transfection in resistant K562/IMA-3 cells. At the final stage of the study, we checked apoptotic case of the cells following miRNA treatments and observed that leukemic cell apoptosis was induced significantly as 5.06-fold in resistant cells.

Overall, uniqueness of our study is handling imatinib resistance phenomenon in much different points of molecular approaches. But there are some reported papers regarding miRNA expressional differences among parental and imatinib resistant cells like our study. In 2012, Turrini E et al. published a paper in the means of discovering the facts about imatinib resistance by comprising 667 miRNAs in array system in parental and low-dose imatinib resistant (4 months of imatinib treatment) K562 cells. They reported interaction between miR-212 and miR-328 with ABCG2 [20].

A more recent study was reported by Li Y et al. in 2013, and they used RNAi approach for selective inhibition of BCRABL expression and then monitored miR-203 expressional changes among parental and imatinib resistant BaF3-BCRABL cells. Overexpression of miR-203 inhibited cell growth and colony formation ability of leukemic cells, whereas targeting BCR-ABL expression by siRNA in correlation with miR-203 mimic transfection restored the sensitivity to imatin$\mathrm{ib}$ in cells expressing the imatinib resistant BCR-ABL kinase domain mutant T315I [21]. Similar to our aim, these two reported studies also put forward the molecular basis of imatinib resistance in the means of miRNA profiling approach combined with siRNA applications.

Our investigations have disclosed the importance of JAK/ STAT pathway member STAT5A in gain of resistance to chemotherapeutic agent imatinib and how silencing resulted in gain of response. Besides STAT5A, also STAT3 has significant roles in hematological malignancies as we and others also have reported before $[22,23]$. Similar to our inferences about the significance of JAK/STAT pathway members - especially STAT family, Rozovski U et al. reported that STAT3 regulated microRNA gene expression in chronic lymphocytic leukemia cells and presence of activated STAT3 had a profound effect on miRNA expression in CLL cells [24]. In another very recently published study, suppressing miR-155 expression through JAK/STAT pathway member STAT3 exhibited a therapeutic approach by promoting human acute myelogenous leukemia cell model HL60 cells' differentiation in accompany of leukemia inhibitory factor receptor $\alpha$-chain (LIFR $\alpha$-CT3) and its TAT fusion protein [25]. All these data exhibit the efficacy of targeting STAT family member-based hematological cancer therapies.

In conclusion, we put a milestone in discovering the molecular basis of gained imatinib resistance due to silencing STAT5A and by monitoring miRNome and transcriptome assays; we highlighted hsa-miR-2278 as a tumor suppressor. Thus, upregulating hsa-miR-2278 expression might be a functional therapeutic approach in order to have clinical benefit and gain of drug response in CML therapy for inhibiting leukemic cell proliferation and inducing apoptosis. In the future, it will be beneficial to analyze differing expression profiles in both CML responder and imatinib-resistant patients.

Acknowledgments This study was financially supported by Ege University Research Fund [grant number APAK 2013-TIP/083] to BTK.

Conflicts of interest None 


\section{References}

1. Deininger MW, Goldman JM, Melo JV. The molecular biology of chronic myeloid leukemia. Blood. 2000;96(10):3343-56.

2. Groffen J, Stephenson JR, Heisterkamp N, de Klein A, Bartram CR, et al. Philadelphia chromosomal breakpoints are clustered within a limited region, bcr, on chromosome 22. Cell. 1984;36:93-9.

3. Lugo TG, Pendergast AM, Muller AJ, Witte ON. Tyrosine kinase activity and transformation potency of bcr-abl oncogene products. Science. 1990;247:1079-82.

4. Druker BJ et al. Effects of a selective inhibitor of the Abl tyrosine kinase on the growth of Bcr-Abl positive cells. Nat Med. 1996;2:561-6.

5. Apperley JF. Part I: mechanisms of resistance to imatinib in chronic myeloid leukaemia. Lancet Oncol. 2007;8:1018-29.

6. Balabanov S, Braig M, Brümmendorf TH. Current aspects in resistance against tyrosine kinase inhibitors in chronic myelogenous leukemia. Drug Discov Today Technol. 2014;11:89-99.

7. Soverini $\mathrm{S}$ et al. Philadelphia-positive patients who already harbor imatinib-resistant Bcr-Abl kinase domain mutations have a higher likelihood of developing additional mutations associated with resistance to second- or third-line tyrosine kinase inhibitors. Blood. 2009;114(10):2168-71.

8. Turhan AG. STAT5 as a CML target: STATinib therapies? Blood. 2011;117(12):3252-3

9. Sillaber C, Gesbert F, Frank DA, Sattler M, Griffin JD. STAT5 activation contributes to growth and viability in Bcr/Abltransformed cells. Blood. 2000;95(6):2118-25.

10. Warsch W, Kollmann K, Eckelhart E, Fajmann S, Cerny-Reiterer S, Holbl A, et al. High STAT5 levels mediate imatinib resistance and indicate disease progression in chronic myeloid leukemia. Blood. 2011;117(12):3409-20.

11. Kosova B, Tezcanli B, Ekiz HA, Cakir Z, Selvi N, Dalmizrak A, et al. Suppression of STAT5A increases chemotherapeutic sensitivity in imatinib-resistant and imatinib-sensitive K562 cells. Leuk Lymphoma. 2010;51(10):1895-901.

12. Borna H, Imani S, Iman M, Azimzadeh Jamalkandi S. Therapeutic face of RNAi: in vivo challenges. Expert Opin Biol Ther. 2015;15(2):269-85.

13. Song SJ, Pandolfi PP. MicroRNAs in the pathogenesis of myelodysplastic syndromes and myeloid leukaemia. Curr Opin Hematol. 2014;21(4):276-82.

14. Wang $\mathrm{H}, \mathrm{Hu} \mathrm{H}$, Zhang Q, Yang Y, Li Y, Hu Y, et al. Dynamic transcriptomes of human myeloid leukemia cells. Genomics. 2013;102:250-6.
15. Lill CM, Schilling M, Ansaloni S, Schröder J, Jaedicke M, Luessi F, et al. Assessment of microRNA-related SNP effects in the 3' untranslated region of the IL22RA2 risk locus in multiple sclerosis. Neurogenetics. 2014;15(2):129-34.

16. Vaz C, Ahmad HM, Bharti R, Pandey P, Kumar L, Kulshreshtha R, et al. Analysis of the microRNA transcriptome and expression of different isomiRs in human peripheral blood mononuclear cells. BMC Res Notes. 2013;28(6):390. doi:10.1186/1756-0500-6-390.

17. Xiong Q, Yang Y, Wang H, Li J, Wang S, Li Y, et al. Characterization of miRNomes in acute and chronic myeloid leukemia cell lines. Genomics Proteomics Bioinformatics. 2014;12(2):79-91.

18. Bhutra S, Lenkala D, LaCroix B, Ye M, Huang RS. Identifying and validating a combined mRNA and MicroRNA signature in response to imatinib treatment in a chronic myeloid leukemia cell line. PLoS One. 2014;9(12), e115003.

19. Bibi S, Arslanhan MD, Langenfeld F, Jeanningros S, CernyReiterer S, Hadzijusufovic E, et al. Co-operating STAT5 and AKT signaling pathways in chronic myeloid leukemia and mastocytosis: possible new targets of therapy. Haematologica. 2014;99(3):417-29.

20. Turrini E, Haenisch S, Laechelt S, Diewock T, Bruhn O, Cascorbi I. MicroRNA profiling in K-562 cells under imatinib treatment: influence of miR-212 and miR-328 on ABCG2 expression. Pharmacogenet Genomics. 2012;22(3):198-205.

21. Li Y, Yuan Y, Tao K, Wang X, Xiao Q, Huang Z, et al. Inhibition of BCR/ABL protein expression by miR-203 sensitizes for imatinib mesylate. PLoS One. 2013;8(4), e61858.

22. Kaymaz BT, Selvi N, Gündüz C, Aktan C, Dalmızrak A, Saydam $\mathrm{G}$, et al. Repression of STAT3, STAT5A, and STAT5B expressions in chronic myelogenous leukemia cell line K-562 with unmodified or chemically modified siRNAs and induction of apoptosis. Ann Hematol. 2013;92(2):151-62.

23. Al Zaid Siddiquee K, Turkson J. STAT3 as a target for inducing apoptosis in solid and hematological tumors. Cell Res. 2008;18(2): 254-67.

24. Rozovski U, Calin GA, Setoyama T, D'Abundo L, Harris DM, Li P, et al. Signal transducer and activator of transcription (STAT)-3 regulates microRNA gene expression in chronic lymphocytic leukemia cells. Mol Cancer. 2013;12:50.

25. Xu S, Xu Z, Liu B, Sun Q, Yang L, Wang J, et al. LIFR $\alpha$-CT3 induces differentiation of a human acute myelogenous leukemia cell line HL-60 by suppressing miR-155 expression through the JAK/STAT pathway. Leuk Res. 2014;38(10):1237-44. 